\title{
Development of a metering system to evaluate power distribution losses
}

\author{
E. A. Silva ${ }^{1}$, O. H. A. Junior ${ }^{2}$, A. D. Spacek ${ }^{1}$, J. D. Spacek ${ }^{3}$, J. M. Neto ${ }^{1}$, L. F. Venturini ${ }^{1}$, V. L. Coelho ${ }^{1}$, \\ A. A. Tavares ${ }^{1}$, M. Sunada ${ }^{1}$, J. C. Cutipa-Luque ${ }^{1}$ \\ ${ }^{1}$ Department of Electrical Engineering \\ SATC Faculty \\ Rua Pascoal Meller, 71, CEP 88805380, Comerciário. Criciúma, SC (Brazil) \\ Phone/Fax number: +5548 34317550/+5548 34317501, e-mail: andre.tavares@ satc.edu.br \\ ${ }^{2}$ Department of Renewable Energies Engineering \\ UNILA, Federal University of Latin American Integration Av. Sílvio Américo Sasdelli, 1842. Foz do Iguaçu, PR (Brazil) \\ Phone/Fax number: +55 45 35292832, e-mail: oswaldo.junior@unila.edu.br \\ 3 Cooperativa Pioneira de Eletrificação - COOPERA \\ Rodovia Josephina Lodetti Vassoler, 2801, CEP 88850-000, Santa Cruz. Forquilhinha, SC (Brazil) \\ e-mail: jefferson@coopera.com.br
}

\begin{abstract}
This work focuses on the development of a metering system that will be used to evaluate power distribution losses. The data information used by the metering system is recorded with digital metering devices, installed over the electrical network. A metering device records some critical information relative to the electrical network and sends it to a workstation via remote wireless communication. The practical issues over implementation are discussed and analyses of precision over standard measurement equipment are presented.
\end{abstract}

\section{Key words}

Power distribution, low voltage, instrumentation, Zigbee, smartgrid.

\section{Introduction}

Losses in electric power systems are classified as technical and non-technical, and are intrinsic in the electricity sector [1]. The losses in electric power systems are the main concern for electricity distribution companies. The investments in developing systems and smart devices to mitigate and identify these technical and non-technical losses have increased recently. The major sources of technical losses in power distribution systems are transformers and power lines [3]. The non-technical losses are mainly caused by theft, non-payment consumers, and error in accounting and registering consumer energy [10].

Nowadays, one of the main challenges for power distribution companies is to establish mitigation procedures to reduce these losses. According to the Brazilian Electricity Regulatory Agency (ANEEL), more than three billion US dollars are spent every year in Brazil due to non-technical losses [2].

The mitigation of losses becomes the distribution system more efficient and increase the capacity of the companies in order to feed new consumer units. The development of a system to identify and minimize losses is important since it will allow electricity distribution companies detect deviations and theft of energy.

The paper presents a prototype of single-phase multifunction meter and register in order to collect data relative to the main parameters of the electric network. Based on the data recorded, a software platform executes algorithms of detection losses. The instrument will also give some critical parameters related to quality of energy.

The organization of the paper is as follows: section 1 presents a brief introduction over power distribution losses; section 2 presents the losses detection systems in which the metering devices will be used; section 3 presents a description of power loss meter; section 4 presents the calibration and results of measurement data. Finally, the conclusions of the paper are presented in section 5 .

\section{Losses Detection System}

In order to detect the electrical losses, the implementation of smart devices on a whole distribution system can demand high investment. Instead, methods and approaches to detection based on minimum use of electronic devices and supported by management software, turn the project less expensive and attractive for companies.

The present work is part of a wide project in course consisting in to develop a system for automatic detection of losses in power distribution system. The project aims to tackle the problem of minimization of power distribution losses that will benefit electricity distribution companies located in the south of Santa Catarina state, Brazil. The main idea is to collect data through multiple measurement devices installed over the grid and to feed management software which determines local of misbehaviour or irregular losses. The probabilistic 
approaches will estimate and identify electrical network misbehaviour, based on data information over typical charge curves of consumers, typical values of technical losses of transformers and power lines [10]. More details of approaches that compute global losses can be found in references [4], [7].

Fig. 1 presents a typical scenario, part of electrical grid, where multiple metering devices are installed in order to collect data. The grid is composed by single-phase consumers and by three-phase consumers, $220 \mathrm{~V}$ and 380 $\mathrm{V}$, respectively. The electric power distribution grid is feed through a three phase transformer connected upstream to medium tension voltage $15 \mathrm{kV}$. The single metering devices (1MDs) are installed for single-phase consumers and three metering devices (3MDs) are installed for threephase consumers.

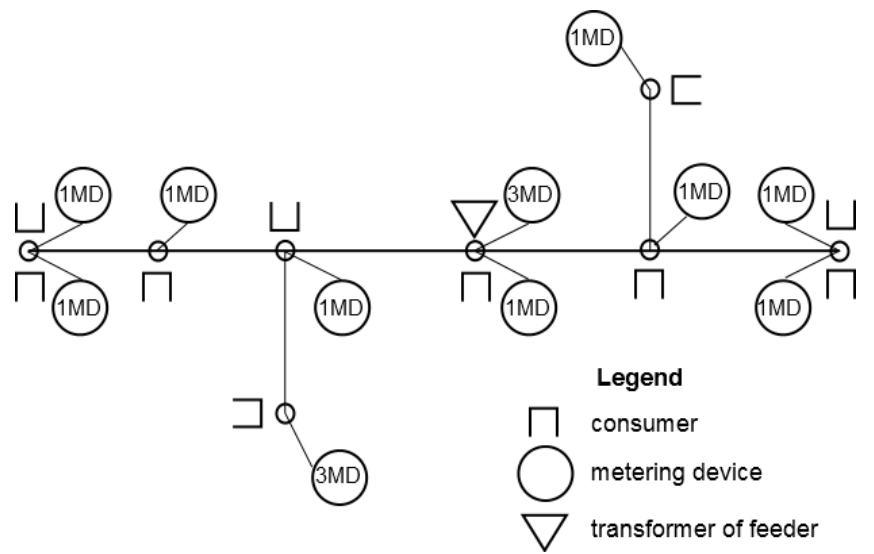

Fig. 1. Allocations of metering devices over an electric power network.

\section{Power loss meter}

The core of the detection system is the metering device which records some critical variables of the grid that will be processed by the system detection losses software. The procedure for hardware development follows a block diagram sequence presented in Figure 2.

The sensors collect the information of current and voltage using a current transformer (CT) and a tension divider technique. The CT has precision of $0.6 \%$, has linearity property of $0.5 \%$, and works with a range of operation between 0.1 to 100 Amperes. The tension divider has precision of $1 \%$ and is unsusceptible to temperature variations due to the inherent compensated circuit embedded into the IC (Integrated Circuit).

In the signal processing stage, the current and tension signals are conditioning, filtered, and processing by an integrated circuit of specific application ADE7753 [5]. The ADE7753, manufactured by Analog Devices, is an integrated circuit for high precision smart meter, has two analog-to-digital converters (ADC) with 16 bits of resolution and with sigma-delta modulation. This IC limits maximum input voltage up to $\pm 1 \mathrm{~V}$ and has programmable gain amplifiers. Using a standard serial peripheral interface (SPI), the IC is flexible to reconfigure its critical parameters commonly required in calibration procedures.
Data processing is treated using the popular Arduino system on module. The microcontroller Atmega328 microcontroller is the core of the metering device which communicates with the specific IC ADE7753 for setting properly its parameters [6].

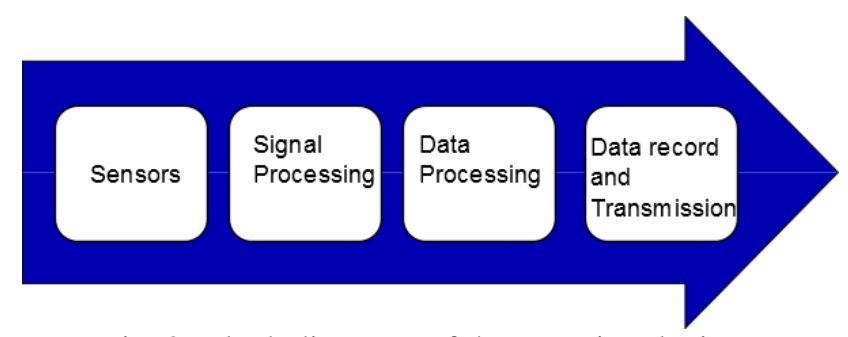

Fig. 2. Block diagrams of the metering device.

Data record and transmission is carried out using a security digital card (SD) memory and an Xbee-pro module. After data processing, the microcontroller saves the data on a SD card and also sends the information via wireless Xbee-pro. The wireless module, Xbee-pro, works at frequency of $2.4 \mathrm{GHz}$, uses the open source Zigbee protocol, is commonly used in control and automation due mainly to its low cost, its low power, and its support for mesh topology (IEEE 802.15.4). A mesh topology has become a suitable characteristic of smart devices because they cover large areas and can be easily adapted over the topology of the power electrical grid [8].

Fig. 3 presents the schematic of the conditioning circuit used to develop metering devices. All components are set according to the specification of datasheets [5]. The J1 and $\mathrm{J} 2$ connectors correspond to the tension and current sensing terminals. The current is sensing using a current transformer.

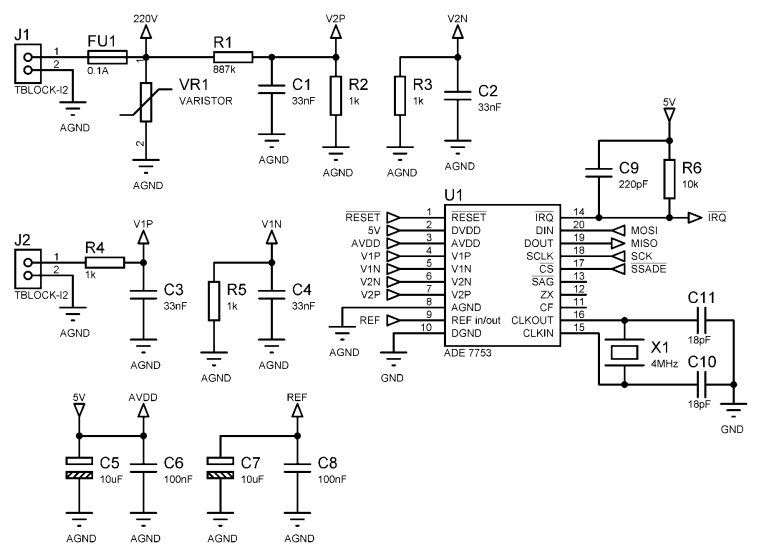

Fig. 3. Conditioning circuit schematic of a metering device.

Fig. 4 presents the PC board in $3 \mathrm{~d}$ view of the metering device (a) and the physical picture of the first prototype (b). The main components are indicated in the legend.

Fig. 5 presents the final result of the metering device with terminals for current and tension sensing. The tension connectors are also used as input voltage for the internal DC source, designed to feed properly electronic circuits of the metering device. 


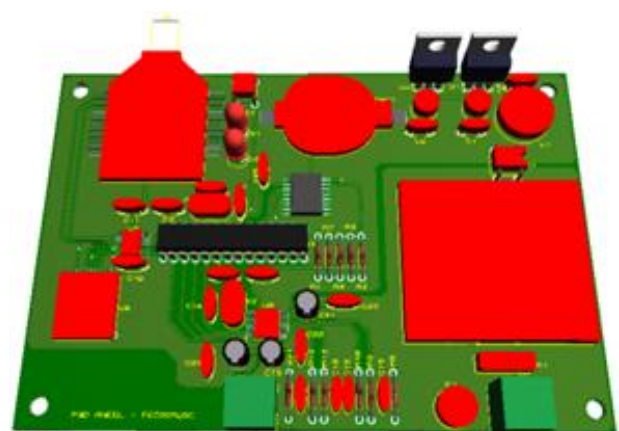

(a)

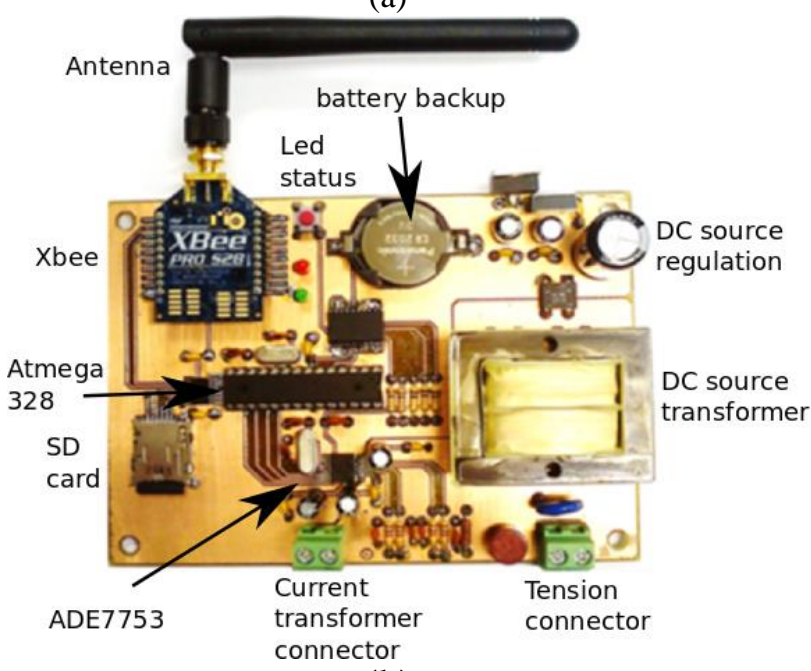

(b)

Fig. 4. Metering device: (a) 3D design of PC Board, (b) physical electronic circuit board.

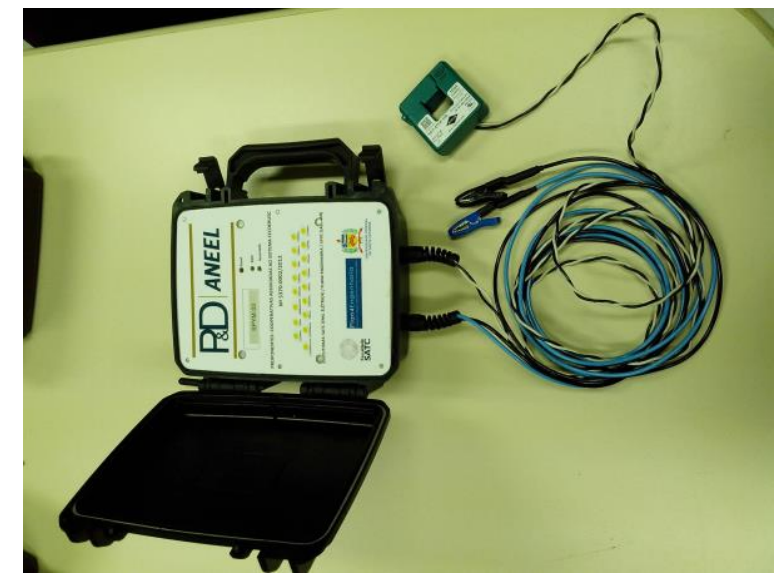

Fig. 5. Photo of the metering device with alligator terminals for tension measurement and with current transformer for current measurement.

\section{Calibration and results}

The metering devices are calibrated using a certified equipment by INMETRO (National Institute of Metrology, Quality and Technology), model 400.3 of Meter Test Equipment AG [9], presented in Figure 6. This equipment is fully automatic, with precision of 0.02 , has an integrated power source.

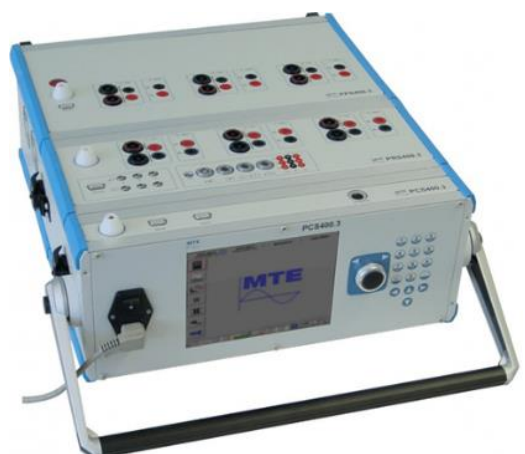

Fig. 6. Meter test equipment AG [9].

Tab. I presents the tension calibration for a new metering device where the maximum error is $0.01 \%$ relatives to nominal $220 \mathrm{~V}$. The maximum error is $0.40 \%$ relatives to tension test of $240 \mathrm{~V}$. Another different values of tension are tested in order to obtain minimal error compararing with the standard MTE equipment.

Tab. II presents the current calibration for a new metering device where the maximum error is $0.40 \%$ relatives to low current $0.50 \mathrm{~A}$. Another different values of current are tested in order to obtain values with minimal error compararing with the standard MTE equipment.

Table I. - Tension calibration

\begin{tabular}{|r|c|c|c|}
\hline \multirow{2}{*}{ Volts (V) } & \multicolumn{3}{|c|}{ Error (\%) } \\
\cline { 2 - 4 } & Minimum & Medium & Maximum \\
\hline 200 & 0.22 & 0.25 & 0.26 \\
\hline 210 & 0.12 & 0.13 & 0.14 \\
\hline 220 & 0.00 & 0.00 & 0.01 \\
\hline 230 & 0.17 & 0.18 & 0.19 \\
\hline 240 & 0.39 & 0.40 & 0.40 \\
\hline
\end{tabular}

Table II. - Current calibration

\begin{tabular}{|r|c|c|c|}
\hline \multirow{2}{*}{ Current (A) } & \multicolumn{3}{|c|}{ Error (\%) } \\
\cline { 2 - 4 } & Minimum & Medium & Maximum \\
\hline 0.50 & 0.41 & 0.41 & 0.41 \\
\hline 5.00 & 0.09 & 0.11 & 0.11 \\
\hline 10.00 & 0.00 & 0.00 & 0.00 \\
\hline 15.00 & 0.09 & 0.10 & 0.10 \\
\hline 20.00 & 0.17 & 0.17 & 0.18 \\
\hline 25.00 & 0.19 & 0.20 & 0.20 \\
\hline
\end{tabular}

The electronic measurement devices have carried out measurements of typical consumers and then were installed on the electric network for technicians of power distribution companies. According to the losses detection methodology, the sampling data are recorded in a period time of 10 days and with 1 minute step time (see Figure 7).

Fig. 7 presents the plot of active energy recorded in a period of time of eight days for a typical consumer. Figure 8 presents the plot of active energy recorded in period time of two days for another typical consumer. The post-processing data system of detection losses is 
flexible allowing select many different period times and different consumers.

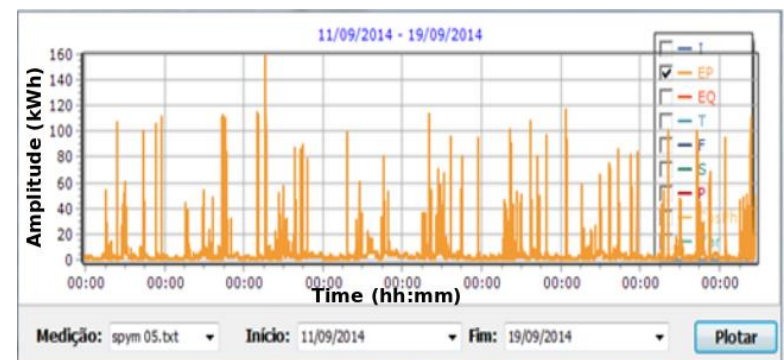

Fig. 7. Active energy (kWh) of a consumer recorded between october $11-19$ of 2014 .

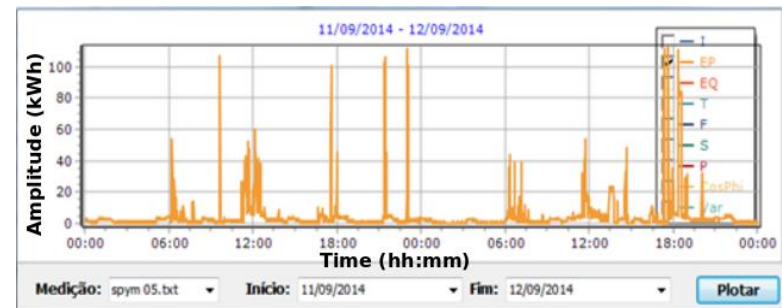

Fig. 8. Active energy (kWh) of a consumer recorded between october $11-12$ of 2014 .

\section{Conclusions}

The paper assessed the development of a metering device as part of a system for evaluation of power distribution losses. The goal of the project is to implement low cost alternatives for power distribution companies located in the south region of Santa Catarina state in Brazil. The metering devices here developed were calibrated and have been used in measurement process giving necessary information for algorithms of detection losses. The measurement devices are tested in low voltage distribution systems and the data collected by consumers are quite good for post-processing procedures. The hardware design of the measurement systems is versatile and many similar instruments can be replicated following the patent guidelines [11].

\section{Acknowledgement}

This Project is being developed under the Coopera R\&D program which is regulated by ANEEL (5370-0002-2012). The authors would like to thank to the Federation of Cooperatives of Energy of Santa Catarina State (FECOERUSC) for supporting and facilities.

\section{References}

[1] Benedict, E.; Collins, T.; Gotham, D.; Hoffman, S.; Karipides, D.; Pekarek, S.; and Ramabhadran, R. Losses in electrical power systems, 1992.

[2] EPE - EMPRESA DE PESQUISA ENERGÉTICA. Demanda de Energia Elétrica (in Portuguese), 2012.

[3] Sun, D.I.H.; Abe, S.; Shoults, R.R.; Chen, M.-S.; Eichenberger, P.; Farris, D. Calculation of Energy Losses in a Distribution System. Power Apparatus and Systems, IEEE Transactions on, vol. PAS-99, no.4, pp. 1347, 1356, July 1980.
[4] ANEEL. Distribution Procedures of Electrical Energy in the Brazilian Electricity Sector - Module 7 (in Portuguese). Normative resolution n 543/2013, April 2013.

[5] ANALOG DEVICES (Massachusetts). Single-Phase Multifunction Metering IC with di/dt Sensor Interface: ADE7753. Norwood, 2010. 60 p. (Rev C).

[6] ATMEL corporation (California). Atmel 8-bit Microcontroller with 4/8/16/32KBytes In-System Programmable Flash. San Jose, 2013. 660 p. (Rev.: 8271GAVR-02/2013).

[7] Bratti, A. C.; Ando Junior, O. H.; Neto, J. M.; Spacek, A. D.; Coelho, V. L.; Tavares, A. A.; Oliveira, M. O. Characterization of losses at the distribution system. International Journal of Automation and Power Engineering (IJAPE), Vol. 2, issue 5, July 2013.

[8] Gungor, V.C.; Sahin, D.; Kocak, T.; Ergut, S.; Buccella, C.; Cecati, C.; Hancke, G.P., "Smart Grid Technologies: Communication Technologies and Standards," Industrial Informatics, IEEE Transactions on , vol.7, no.4, pp.529,539, Nov. 2011.

[9] MTE. Meter test equipment AG. Datasheets of Meter Test Equipment: PTS 400.3, class 0.02 Modular three-phase Portable Test System, Switzerland, 2012.15 p.

[10] Neto E.A.C.A.; Coelho J. Probabilistic methodology for Technical and Non-Technical Losses estimation in distribution system. Electric Power Systems Research. Volume 97, April 2013, Pages 93-99, ISSN 0378-7796.

[11] Patent number protocol BR1020140061126. Measurement system of power distribution losses in two layers (in Portuguese). Brazilian National Institute for Intellectual Properties. 2014. 